\title{
The Sporulation of Clostridium tetani
}

\author{
By K. A. BISSET \\ Department of Bacteriology, University of Birmingham
}

SUMMARY: In the sporulation of Clostridium tetani, a rod-like fusion nucleus is formed from the two nuclear units typical of a 'smooth' bacillus. The fusion nucleus divides into two smaller rods, one of which degenerates; the other is included in the spore. The nucleus remains rod-shaped until the maturation of the spore is almost complete, when it is transformed into a spherical, eccentric spore nucleus.

The cytology of sporulation in certain types of bacillus is well known (Badian, 1933; Klieneberger-Nobel, 1945; Flewett, 1948), and it is generally agreed that the four nuclear units of the bacillus form a rod-shaped fusion nucleus, from which, usually after a cell division, the four units are regenerated. One unit is enclosed in the spore and the other three are rejected.

In my experience this scheme is applicable only to bacilli of 'rough' morphology, which are multicellular and have one nuclear unit in each cell, and not to those of 'smooth' morphology, which possess two nuclear units in each unicellular bacillus (Bisset, 1948).

The present study describes the sporulation of Clostridium tetani, a species which is normally 'smooth', and which also differs from the species previously described in producing a very large terminal spore. The large size of the spore enabled the behaviour of its nuclear material, during maturation, to be observed with reasonable clarity.

\section{MATERIALS AND METHODS}

Two strains of $\mathrm{Cl}$. tetani were used. One was isolated from a fatal case of tetanus in man, the other from horse-dung. They were grown anaerobically upon pepsinized blood-agar, at $37^{\circ}$. Preparations were stained by the acidGiemsa technique.

\section{Observations}

Sporulation was well advanced in cultures $24 \mathrm{hr}$. old (Pl. 1, figs. 1, 2). Vegetative cells contained the two pairs of chromosomes typical of the smooth morphology (Fig. 1a). The formation of the fusion nucleus (Fig. 1c) was preceded by a stage in which an irregular, broken rod was formed (Fig. 1b). The fusion nucleus then assumed its compact rod shape, and divided into two (Fig. 1d). Up to this point the process was exactly comparable with that described by Klieneberger-Nobel; but thereafter was markedly different. The division of the nuclear rod was not followed by division of the bacillus, which remained entire. One of the daughter rods retained its form, and became the nucleus of the spore, the other became spherical in form, and then disintegrated (Pl. 1, fig. 3; and Fig. $1 e, f$ ). The granular remains of this disintegrated nuclear unit were often seen in bacilli containing young spores (Pl. 1, fig. 2). 
The remaining nuclear unit retained its rod shape, and was surrounded by a sharply bounded, refractive area which increased in size until it completely filled the swollen extremity of the bacillus (Pl. 1, fig. 2; and Fig. $1 f, g, h$ ). As the spore matured, the nuclear body was transformed from a rod to an eccentric, spherical body (Pl. 1, figs. 2, 3; and Fig. $1 h, i, j$ ).

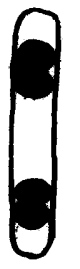

$\boldsymbol{a}$

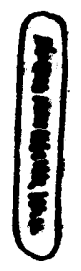

b

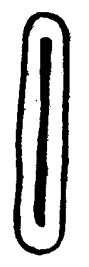

c

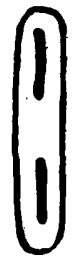

d

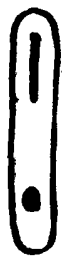

e

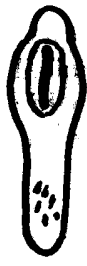

$f$

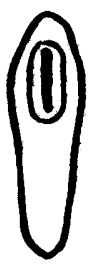

$\mathbf{g}$
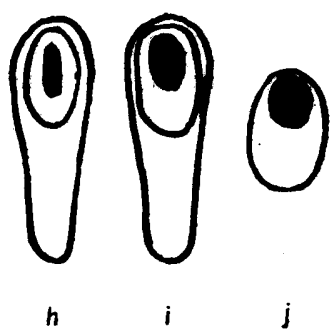

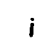
$j$

Fig. 1. Sporulation in $\mathbf{C l}$. tetani.

\section{DISCUSSION}

In the sporulation of $\mathrm{Cl}$. tetani it appears that the formation of a rod-shaped fusion nucleus, a short-lived diploid stage, is followed by a reduction division, in which half the nuclear material is rejected. This process differs from that described by several authors as occurring in bacilli of 'rough' morphology, in which a cell division may precede sporulation, and in which three out of four nuclear units are rejected. It differs also in that the discarded nuclear material is absorbed into the cytoplasm, whereas in the 'rough' forms of other species its disappearance accompanies the dissolution of the bacillus and release of the spore.

The behaviour of the spore nucleus during maturation is of interest. The retention of the rod shape within the almost spherical spore may be taken to indicate that this nuclear form is not simply that imposed upon a mass of nuclear material lying within a rod-shaped cell, but that it possesses some structural coherence. The drumstick appearance of the sporing bacillus, which is not seen in water-mounted preparations, is presumably an artefact due to drying.

\section{REFERENCES}

Badian, J. (1938). Eine cytologische Untersuchung über das Chromatin und den Entwicklungszyklus der Bakterien. Arch. Mikrobiol. 4, 409.

Bisset, K. A. (1948). The cytology of smooth and rough variation in bacteria. J. gen. Microbiol. 2, 83.

FueweTt, T. H. (1948). Nuclear changes in Bacillus anthracis and their relation to variants. J. gen. Microbiol. 2, 325.

KunENEBERGER-Nobel, E. (1945). Changes in the nuclear structure of bacteria, particularly during spore formation. J. Hyg., Camb., 44, 99. 
Journal of General Microbiology, Vol. 4, No. 1

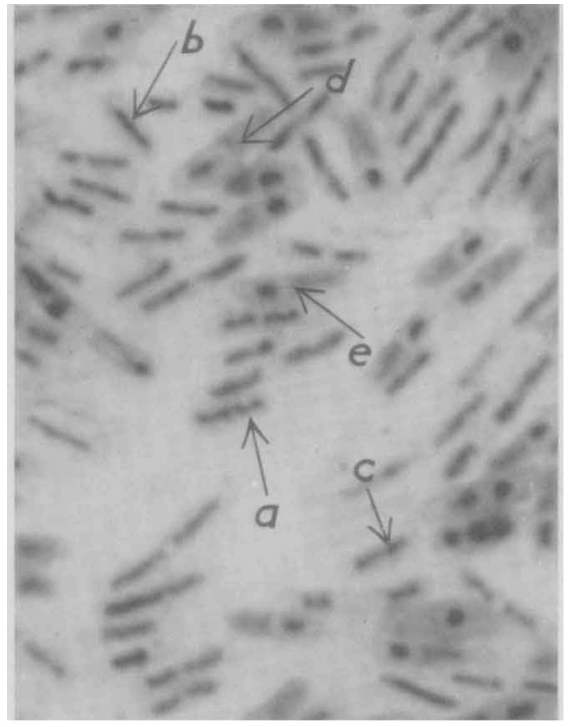

Fig. 1

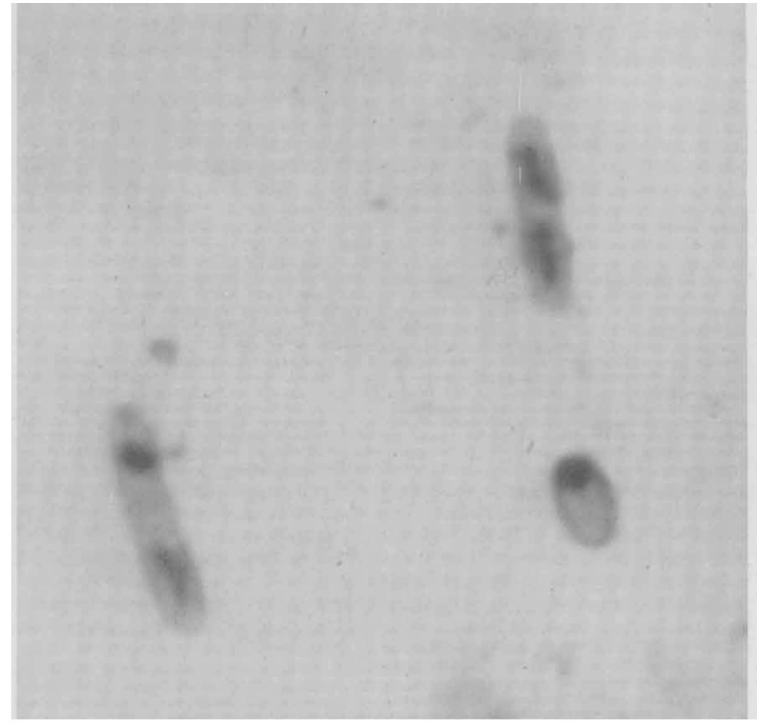

Fig. 3

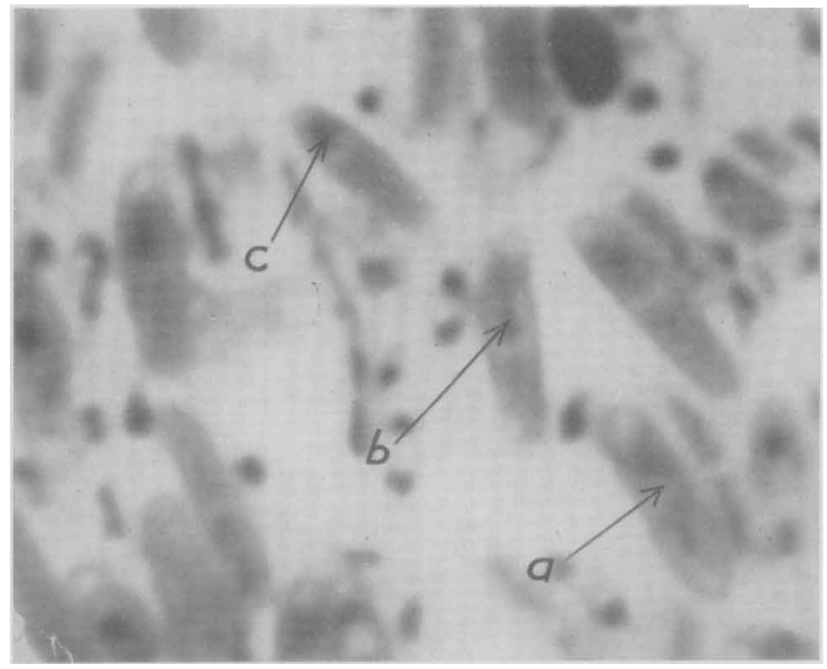

Fig. 2

K. A. Bisset-The sporulation of Cl. tetani. Plate 1 


\section{EXPLANATION OF PLATE}

Fig. 1. Cl. tetani, vegetative cells and stages in sporulation. $\times 3000$. (a) Vegetative cell; (b) fusion nucleus; $(c)$ fusion nucleus after division; $(d)$ early stage of spore formation; (e) later stage of spore formation.

Fig. 2. Cl. tetani, stages in sporulation. $\times 6000 .(a)$ Enclosure of one nucleoid in spore, degeneration of the other; (b) early spore containing rod-shaped nucleus; $(c)$ later spore, containing spherical nucleus.

Fig. 3. Cl. tetani, one cell showing divided fusion nucleus, the other showing half of the nucleus commencing to degenerate. Also a mature spore. $\times 6000$.

(Received 18 February 1949) 